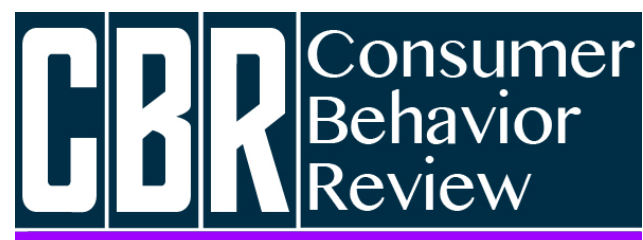

Revista Comportamento do Consumidor
Batista, K., Araújo, M. R. M., Lima, L. G. B., \& Martins, I. M. (2021). Análise da Intenção de uso de Fintechs a partir da Adaptação do Modelo UTAUT2. Consumer Behavior Review, 5(1), 77-88.
ISSN: 2526-7884

Editor: Prof. Dr. Marconi Freitas da Costa

E-mail: cbr@ufpe.br
Avaliação: Double blind review

Recebido: 20 de maio, 2020

Aceito: 30 de Novembro, 2020

\title{
ANÁLISE DA INTENÇÃO DE USO DE FINTECHS A PARTIR DA ADAPTAÇÃO DO MODELO UTAUT2
}

\author{
Analysis of the intention to use fintechs based on the adaptation of the UTAUT2 model
}

Karen Batista ${ }^{1}$

ORCID: http://orcid.org/0000-0002-4918-6219

E-mail: karen.batista@hotmail.com

Marley Rosana Melo de Araújo ${ }^{1}$

ORCID: http://orcid.org/0000-0002-5161-6863

E-mail: marleymeloaraujo@gmail.com

Lucas Gabriel Bezerra Lima ${ }^{1}$

ORCID: http://orcid.org/0000-0003-2116-1850

E-mail: limalucasgabrielbezerra@gmail.com

Ingrid de Matos Martins ${ }^{1}$

ORCID: http://orcid.org/0000-0001-7708-4087

E-mail: iingrindmartins@hotmail.com

${ }^{1}$ Universidade Federal de Sergipe, São Cristovão, Brasil

\section{Resumo}

Esse estudo teve como objetivo analisar a intenção de uso de fintechs por meio de variáveis propostas por Venkatesh, Tong e Xu (2012) e Slade et al. (2015). Para tanto, foi realizado um survey, com uma amostra não probabilística de 106 respondentes. Os dados foram analisados por meio de testes como correlação de Pearson e Spearman, teste T e regressão múltipla padrão, no software SPSS,

\begin{abstract}
This study aimed to analyze the intention to use fintechs through variables proposed by Venkatesh, Tong and Xu (2012) and Slade et al. (2015). For this, a survey was carried out, with a non-probabilistic sample of 106 respondents. Data were analyzed using tests such as Pearson and Spearman correlation, $\mathrm{t}$ test and standard multiple regression, using SPSS software, version 20. As a result, only
\end{abstract}


versão 20. Como resultados, apenas a expectativa de desempenho, valor de preço e hábito afetaram a intenção de uso de fintech. Desse modo, cabe às empresas de fintech investir no aprimoramento das suas funcionalidades, a fim de aumentar a expectativa de desempenho, melhorar o custobenefício e influenciar hábito de utilizar fintech, investindo em ações de marketing, dando incentivos aos consumidores e gerenciando o seu relacionamento com esses. Palavras-chave: Fintechs; Intenção de Uso; Regressão Múltipla. the expected performance, price value and habit affected the intention to use fintech. Thus, it is up to fintech companies to invest in improving their functionalities, in order to increase performance expectations, improve cost-benefit and influence the habit of using fintech, investing in marketing actions, giving incentives to consumers and managing their relationship with those.

Keywords: Fintech; Intention to Use; Multiple Regression.

This work is licensed under a Creative Commons Attribution 4.0 International License.

\section{INTRODUÇÃO}

Em uma economia capitalista, oligopólio designa a situação de um mercado de certa mercadoria ou serviço contido por pequenos números de produtores e dominantes do mercado que concorrem entre si, cada um forte o suficiente para influenciar preços e quantidade de produtos, de modo a maximizar lucros (Ministério da Fazenda, 2016). Todavia, esse tipo de mercado reduz a concorrência e deixa a oferta do serviço na responsabilidade de poucos, fazendo com que os preços subam e fiquem acima da média, já que concorrem somente entre si (Miranda, 2011).

No Brasil, desde muito tempo, a situação real do mercado financeiro constata o poder bancário nas mãos de poucos bancos, sendo eles detentores de 80\% do Market Share (Finnovation, 2018). No entanto, o setor financeiro vem passando por mudanças significativas devido ao fortalecimento de startups financeiras. Startups são novas empresas de alta tecnologia que se utilizam de Pesquisa e Desenvolvimento (P\&D) num mercado de extrema precariedade, alcançando posições no mercado e em pouca velocidade, tendo em seu escopo uma curva de desenvolvimento que só tende a crescer (Ferreira et al., 2017).

Por meio do fortalecimento tecnológico de startups no mercado financeiro - as fintechs -, uma revolução nesse mercado já estava acontecendo no mundo ao longo dos anos (Arner, Barberis, \& Buckley, 2015). Para o Banco Mundial (2018) a facilidade do smartphone e do acesso à internet por meio do dispositivo, tem acelerado a inclusão financeira a nível global. Segundo Rodrigues (2012, p. 5), os clientes bancários têm utilizado cada vez mais "os meios virtuais internet e mobile banking do que os canais tradicionais para fazer operações financeiras." Nesse sentido, com esse crescimento tecnológico, o mercado mobile e financeiro no Brasil foram impulsionados e contaram com o favorecimento da ampliação do cenário econômico para a população de clientes.

Na sociedade contemporânea, o uso de novas tecnologias já é tratado de forma estratégica pelas instituições, principalmente as de caráter financeiro que hoje desenvolvem meios de conversão digital através da interface do mobile banking, ampliação seu alcance de clientes e reduzindo os processos para contratação e utilização dos produtos disponíveis. No entanto, algumas pessoas ainda resistem em usar a inovação (Baganzi \& Lau, 2017). Soma-se a isso o risco percebido pelos usuários de perder ativos financeiros (Osei-Assibey, 2015).

De acordo com Lisboa e Mendonça (2017) as grandes instituições bancárias são bastante burocráticas para a realização de seus serviços, enquanto que as fintechs surgem no intuito de facilitar os processos por meio do simples uso do aplicativo. Outro ponto muito importante de destaque é que as fintechs usam como estratégia de mercado a prática de taxa de juros bem inferiores em relação ao mercado tradicional, fazendo com que o usuário se sinta mais atraído pelos benefícios (Lisboa \& Mendonça, 2017). 
Diante de tais mudanças no mercado com o surgimento das fintechs como uma inovação no setor, é indispensável conhecer a percepção dos usuários sobre os serviços que estas empresas prestam, para que assim possam adequá-los as expectativas e necessidades dos clientes. Esse trabalho se propôs a analisar a intenção de uso de fintech por meio de variáveis propostas por Venkatesh, Tong e Xu (2012) e Slade et al. (2015). Especificamente pretende-se identificar dentre tais variáveis quais podem exercer alguma influência na intenção de uso de fintechs.

Este artigo encontra-se dividido em cinco partes: a primeira corresponde a esta introdução; em seguida faz-se uma breve apresentação das fintechs; a terceira parte corresponde a metodologia, onde são apresentados os procedimentos metodológicos utilizados nessa pesquisa; a quarta parte apresenta os resultados encontrados; e por fim, a quinta parte são as considerações finais.

\section{FINTECHS}

Os serviços bancários móveis são, antes de tudo, um fenômeno da mobilidade, portabilidade e convergência digital na era virtual. Convergência de tecnologias, de plataformas, de dispositivos, de modelo de negócio e de indústrias. Vários segmentos de alguns setores da economia estão ou serão envolvidos para a consecução de seus objetivos convergentes, sendo que os setores bancários e de telecomunicações já possuem papéis de destaque (Cernev, 2010).

As fintechs podem ser conceituadas como startups que geram inovações no campo do mercado de serviços financeiros trazendo em seu corpo tecnologias que tornam o mercado seus sistemas mais desenvolvidos. Esses startups de finanças, geralmente, são desenvolvidos com o intuito de romper com os complexos do modelo de mercado financeiro, principalmente no Brasil onde predomina o mercado Oligopólio (Conexão Fintech, 2017).

Em um estudo bibliográfico e documental, Ferreira et al. (2017) abordam que nos dois primeiros meses do ano de 2017 mais de duas mil agências bancárias se tornaram inexistentes deixando especulações acerca da crise, e outras acerca do desenvolvimento tecnológico e empreendedor como a oferta das fintechs, no mercado financeiro, porém, os bancos continuam sendo os detentores da moeda e do poder mercadológico, necessitando maior acréscimo de estudos, análises, P\&D para alavancar essas startups no mercado e consolidá-las.

0 fenômeno Fintech tem provocado nos bancos dominadores e nas instituições financeiras tradicionais a necessidade de buscarem modernizações em seus processos, serviços, desenvolvendo novas estratégias de fidelizar clientes para que possam se manter firmes no mercado sem perder seus clientes, se não totalmente, mas em alguns produtos (Ciab Febraban, 2017; Ferreira et al., 2017). As inovações que as fintechs trazem têm permitido a pessoas que não utilizavam bancos tradicionais o acesso a serviços financeiros, por meio de dispositivos móveis (Senyo \& Osabutey, 2020).

Estrategicamente, as fintechs almejam imergir com novos serviços financeiros por meio de seus aplicativos para celulares, sendo muitas vezes mais didáticos, transparecendo mais facilidade de uso, enquanto as grandes instituições bancárias tradicionais são muito burocráticas em suas operações e contratação de um serviço (Finnovation, 2018). 0 ano de 2017 foi um ano importante para as fintechs no Brasil. Nos últimos anos, diversas startups fintech nasceram e em 2017 elas amadureceram, forte 0 suficiente para buscar clientes (Finnovation, 2018).

Em maio de 2018 a Finnovation, juntamente com o Finnovista e o Banco interamericano de Desenvolvimento realizaram um levantamento sobre o número de fintechs no Brasil, e o resultado mostrou que os segmentos com maior número de fintechs são: pagamentos e remessas (com a maior quantidade), empréstimos, gestão financeira empresariais e pessoais e crowfunding imobiliário. Este mapeamento mostrou que a área de investimentos tem muito potencial no ambiente financeiro digital, porém esse segmento ainda é amplamente dominado pelos cinco bancos do oligopólio, no qual detêm 80\% do Market Share em todo Brasil (Finnovation, 2018).

Vários estudos científicos têm sido desenvolvidos nos últimos anos, como os realizados por Marcon (2018) e Araujo (2018) que buscaram entender qual o novo papel das instituições bancárias e quais as ameaças para as mesmas com o advento da revolução fintech. Para isso, utilizaram, respectivamente a realização de um painel com especialistas sobre o tema e da leitura de relatórios das empresas de consultoria financeira. Um cenário bastante relevante foi levantado por Guidi (2018), 
no qual analisou o panorama do cruzamento do cenário entre fintechs e segmentação de clientes que, para facilitar a análise de dados, utilizou o crédito pessoal e seu índice de aprovações e conversões de contratos. $\mathrm{O}$ autor sugere que, através desse estudo, é possível que as instituições do segmento financeiro, seja bancos ou as startups, identifiquem, tendências e trabalhem suas mídias para atração de clientes e superar a concorrência.

Chihimi (2018) realizou uma pesquisa com objetivos semelhantes a essa. Em seu estudo, a autora utilizou o mercado Espanhol para analisar se o advento das fintechs geram disrupção ou evolução no mercado bancário. Para isso, realizou uma triangulação da percepção dos stakeholders, sendo eles: bancários, clientes e fintechs. 0 resultado mostrou que a revolução gera evolução no setor bancário.

Dessa forma, além de ser um campo novo de estudo, existem poucas referências sobre os ares que permeiam essa iniciativa, uma vez que os estudos sobre as fintechs preocuparam-se muito em entender as transformações operacionais internas e a necessidade de mudanças nas estratégias das empresas já atuantes. Analisando a partir da ótica dos clientes é possível compreender como ocorre a escolha por utilizar as fintechs e em que aspectos a experiência pode ser melhorada.

Senyo e Osabutey (2020) realizaram um estudo utilizando uma das inovações das fintechs, o dinheiro móvel, a partir da Teoria Unificada de Aceitação e Uso de Tecnologia 2 (UTAUT2). Os resultados da sua pesquisa demonstraram que a expectativa de desempenho e a expectativa de esforço tiveram efeito na intenção de usar o dinheiro móvel. Contudo, as variáveis valor de preço, motivação hedônica, influência social e percepção de risco não influenciaram a intenção de usar dinheiro móvel. No presente estudo, utilizou-se abordagem semelhante, ao analisar a intenção de uso de fintechs por meio da teoria UTAUT2, com a adição de outras variáveis encontradas na literatura. Os detalhes sobre o modelo utilizado são apresentados no tópico seguinte, bem como os procedimentos metodológicos utilizados nessa pesquisa.

\section{PROCEDIMENTOS METODOLÓGICOS}

No que concerne aos métodos e procedimentos metodológicos, esta pesquisa é classificada como quantitativa, considerando que os resultados e conclusões são baseados na tradução de números em opiniões por meio do uso de recursos e técnicas estatísticas. Quanto aos procedimentos técnicos para o levantamento dos dados necessários para a realização da pesquisa, foi realizado um survey. No que diz respeito à amostragem, foi utilizada a não-probabilista, uma vez que a representatividade probabilística exata da população é inviável, e para isso, foi utilizado o método por conveniência, ou seja, de acordo com a disponibilidade para responder.

Em relação ao instrumento de coleta de dados, foi utilizado um questionário elaborado pelos autores. Nele foram utilizadas perguntas abertas e fechadas, resultando em um total de 54 perguntas. Destas, 5 eram do primeiro tipo (abertas) e as restantes eram fechadas. 0 questionário foi dividido em duas partes: a primeira contendo questões sociodemográficas e questões comportamentais, especificamente sobre uso de fintechs, de internet e sobre compra online; a segunda parte contendo questões sobre o fenômeno, que foram traduzidas dos roteiros de Venkatesh et al. (2012) e Slade et al. (2015). Portanto, como base para esta pesquisa foi utilizado o modelo UTAUT2, desenvolvido por Venkatesh et al. (2012), com adição das variáveis propostas por Slade et al. (2015). A Figura 1 apresenta graficamente as relações testadas nesse estudo.

No modelo UTAU2, a expectativa de desempenho, expectativa de esforço, influência social, condições facilitadoras, motivações hedônicas, valor de preço e hábito, afetam a intenção de uso das fintechs, com as variáveis idade, sexo e experiência sendo moderadoras dessas relações. No entanto, optou-se por não utilizar as relações de moderação e acrescentar outras variáveis propostas por Slade et al. (2015): Inovatividade, Risco Percebido e Confiança. A Tabela 1 a seguir apresenta os fatores e suas definições. 


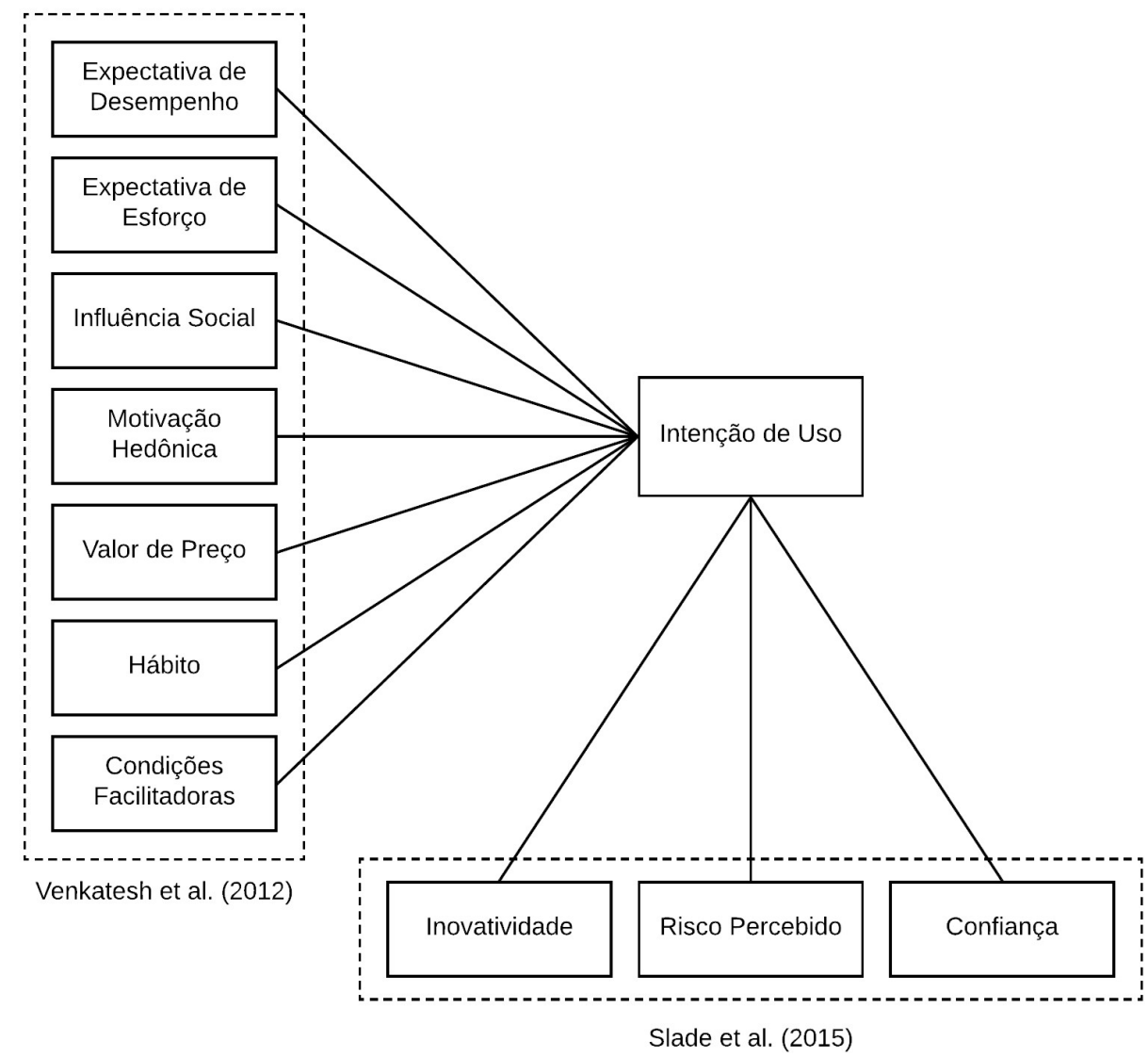

Nota. Fonte: Elaborado pelos autores (2020).

Figura 1. Relações entre os construtos testadas

Tabela 1

Definição dos fatores da pesquisa

\begin{tabular}{|c|c|}
\hline FATOR & DEFINIÇÃO \\
\hline $\begin{array}{l}\text { Expectativa de } \\
\text { Desempenho }\end{array}$ & $\begin{array}{l}\text { Grau em que o indivíduo acredita que, utilizando fintech, essa potencializará seu } \\
\text { desempenho na execução de alguma tarefa. }\end{array}$ \\
\hline $\begin{array}{l}\text { Expectativa de } \\
\text { Esforço }\end{array}$ & Grau de facilidade que o indivíduo associa à utilização de fintechs. \\
\hline Influência Social & $\begin{array}{l}\text { Grau em que o indivíduo acredita que os outros indivíduos consideram importante } \\
\text { que a fintech seja utilizada. }\end{array}$ \\
\hline $\begin{array}{c}\text { Condições } \\
\text { Facilitadoras }\end{array}$ & $\begin{array}{l}\text { Grau em que o indivíduo acredita que o ambiente oferece suporte à utilização de } \\
\text { fintech. }\end{array}$ \\
\hline $\begin{array}{l}\text { Motivação } \\
\text { Hedônica }\end{array}$ & Refere-se à diversão e/ou ao prazer proporcionado ao indivíduo ao usar fintechs. \\
\hline Valor de Preço & $\begin{array}{l}\text { Refere-se à troca aos benefícios percebidos em relação ao custo monetário para usar } \\
\text { fintech. }\end{array}$ \\
\hline Hábito & $\begin{array}{l}\text { Refere-se ao automatismo criado pela aprendizagem de algo, que cria uma } \\
\text { preferência pelo uso da fintech. }\end{array}$ \\
\hline $\begin{array}{c}\text { Intenção } \\
\text { Comportamental }\end{array}$ & Refere-se à intenção de consumir fintechs, não ao uso em si. \\
\hline Inovatividade & $\begin{array}{l}\text { Refere-se ao grau em que o indivíduo está aberto a experimentar novas tecnologias } \\
\text { (fintechs). }\end{array}$ \\
\hline Risco Percebido & $\begin{array}{l}\text { Refere-se a sentimentos de incerteza ou ansiedade sobre o comportamento e a } \\
\text { gravidade ou importância dos possíveis resultados negativos desse comportamento. }\end{array}$ \\
\hline Confiança & Refere-se a uma crença subjetiva de que uma parte cumprirá as suas obrigações. \\
\hline
\end{tabular}

Nota. Fonte: Adaptado de Venkatesh et al. (2012) e Slade et al. (2015). 
Como opções de respostas utilizou-se a escala Likert com sete pontos, pois se torna ideal para mensurar sobre o nível de concordância com os fatores indicados (Likert, 1932). Sanches et al. (2011) mencionam que as escalas de Likert requerem que os entrevistados indiquem seu grau de concordância ou discordância com declarações relativas à opinião ou atitude que está sendo medida. As questões foram mensuradas a partir de uma escala Likert de 7 pontos, onde 1 representava discordo totalmente e 7 representava concordo totalmente.

A coleta de dados foi realizada por meio da plataforma formulários Google, onde foi hospedado o instrumento de coleta dessa pesquisa. 0 mesmo teve o link enviado via Whatsapp, e-mail e outras redes sociais como o Facebook, para diversas pessoas. Ao total foram obtidas 106 respostas válidas. Como instrumento de análise foi utilizado o software SPSS, versão 20. As análises foram realizadas por meio dos testes estatísticos de correlação, teste $\mathrm{T}$ e regressão múltipla, que serão apresentadas no tópico seguinte.

\section{APRESENTAÇÃO E DISCUSSÃO DOS RESULTADOS}

A amostra desse estudo foi composta por 106 respondentes. Destes, 52,8\% são mulheres e $47,2 \%$ são homens. Com relação à idade: 38,7\% têm entre 18 e 25 anos, 39,6\% têm entre 26 e 33 anos, $15,1 \%$ têm entre 34 e 41 anos, e 6,6\% têm 42 anos ou mais. No que diz respeito à renda: 39,6\% têm renda até $\mathrm{R} \$ 1.000,00 ; 29,2 \%$ têm renda entre $\mathrm{R} \$ 1.001,00$ e $\mathrm{R} \$ 2.000,00 ; 15,1 \%$ têm renda entre $\mathrm{R} \$$ $2.001,00$ e $\mathrm{R} \$ 3.000,00 ; 5,7 \%$ têm renda entre $\mathrm{R} \$ 3.001,00$ e $\mathrm{R} \$ 4.000,00 ; 6,6 \%$ têm renda entre $\mathrm{R} \$$ $4.001,00$ e $\mathrm{R} \$ 5.000,00$; e 3,8\% têm renda de $\mathrm{R} \$ 5.001,00$ ou mais. Com relação ao grau de escolaridade dos respondentes da amostra: $49,1 \%$ têm ensino superior incompleto ou menos; $29,2 \%$ têm superior completo; 21,7\% têm pós-graduação.

Com relação ao tempo de uso de fintech, 32,5\% usam entre 2 a 20 meses, 41,5\% usam entre 21 a 41 meses, 14,2\% usam entre 42 a 62 meses, 2,8\% usam há 63 ou mais meses. Sobre a frequência de uso de fintech, 20,8\% usam de 1 a 5 vezes por ano, 14,3\% usam de 6 a 10 vezes por ano, 13,6\% usam de 11 a 15 vezes por ano, 6,5\% usam de 16 a 20 vezes por anos, e $42,9 \%$ usam mais de 20 vezes por ano. No que diz respeito à compra online, $96,2 \%$ afirmaram que compram online, e apenas 3,8\% não compram online. Com relação à frequência de compra online, 38,2\% compram de 1 a 5 vezes por ano, $28,4 \%$ compram de 6 a 10 vezes por ano, 11,8\% compram de 11 a 15 vezes por ano, e 16,7\% compram mais de 20 vezes por ano. Quanto à preferência de compra, 64,2\% preferem comprar online e 35,8\% preferem comprar presencialmente.

Quanto ao tempo de uso de internet, 2,8\% usam há menos de 1 ano, 6,6\% usam de 1 a 5 anos, $30,2 \%$ usam de 6 a 10 anos, 25,5\% usam de 11 a 15 anos, e 34,9\% usam há mais de 15 anos. Com relação à frequência de uso de internet diária, 2,8\% usam por até 1 hora por dia, 5,7\% usam entre 1 e 2 horas por dia, 13,2\% usam entre 2 e 3 horas por dia, 9,4\% usam entre 3 e 4 horas por dia, e 68,9\% usam por mais de 4 horas por dia. No tocante aos dispositivos que utilizam para acessar a internet, $67,9 \%$ utilizam celular e $32,1 \%$ utilizam computador. Sobre o uso de internet banking, $83 \%$ utilizam e $17 \%$ não utilizam.

0 primeiro teste realizado foi uma correlação de Pearson a fim de identificar se existem relações entre as variáveis idade, renda individual mensal e tempo de uso de fintech com os fatores analisados. Como pode-se observar na tabela 2, a variável idade: obteve correlação moderada com a variável renda individual mensal $(r=0,41 ; \mathrm{p}<0,001)$, sugerindo que quanto maior a idade, maior a renda individual mensal para essa amostra; obteve correlação fraca com o tempo de uso de fintech ( $r=$ 0,$23 ; \mathrm{p}=0,049$ ) sugerindo que quanto maior a idade maior o tempo de uso de fintech nessa amostra; e obteve correlação fraca e negativa com a intenção de uso de fintech $(r=-0,22 ; \mathrm{p}=0,022)$ sugerindo que quanto menor a idade maior a intenção de usar fintech. A idade não obteve correlação significativa com nenhum dos fatores. A variável renda individual mensal obteve correlação fraca com o fator motivação hedônica $(r=0,19 ; \mathrm{p}=0,047)$ indicando que quanto maior a renda maior a diversão e o prazer relacionado ao uso de fintech. Também observou-se correlação fraca entre renda individual mensal e confiança $(r=0,23 ; \mathrm{p}=0,018)$, o que sugere que quanto maior a renda maior a confiança em utilizar fintech. 
Quanto aos fatores e sua relação com a intenção de uso de fintech, apenas o fator risco percebido não obteve correlação, enquanto os demais obtiveram correlações de moderada a fraca. Observaram-se correlações fortes entre a intenção de uso de fintech e: hábito $(r=0,74 ; \mathrm{p}<0,001)$; valor de preço $(r=0,74 ; \mathrm{p}<0,001)$; e expectativa de desempenho $(r=0,70 ; \mathrm{p}<0,001)$. Tais resultados indicam que: quanto maior o hábito em usar fintech maior será a intenção de usá-la; quanto maior o custo-benefício de usar fintech maior será a intenção de usá-la; e quanto maior a expectativa de que a fintech potencializará o desempenho de determinadas tarefas, maior a intenção de usá-la.

Também foram relevantes as correlações moderadas entre a intenção de uso de fintech e: expectativa de esforço $(r=0,67 ; \mathrm{p}<0,001)$; motivação hedônica $(r=0,66$; p $<0,001)$; e condições facilitadoras $(r=0,62 ; \mathrm{p}<0,001)$. Deste modo, quanto maior a facilidade que o indivíduo possui em usar fintech, maior será a intenção de usá-la; quanto maior o prazer e diversão associadas ao uso de fintech, maior será a intenção de usá-la; e, quanto mais condições facilitadoras do uso de fintech o indivíduo tem, maior será a intenção de usá-la. A seguir, a tabela 2 demonstra os resultados encontrados no teste correlação de Pearson, cujos resultados significativos estão destacados.

Tabela 2

Correlação de Pearson

\begin{tabular}{|c|c|c|c|c|}
\hline & Idade & $\begin{array}{c}\text { Renda Individual } \\
\text { Mensal }\end{array}$ & $\begin{array}{c}\text { Tempo de uso } \\
\text { de Fintech }\end{array}$ & $\begin{array}{c}\text { Intenção de } \\
\text { uso de Fintech } \\
\end{array}$ \\
\hline Idade & 1 & & & $\begin{array}{c}r=-0,222 \\
p=0,022\end{array}$ \\
\hline Renda Individual Mensal & $\begin{array}{l}r=0,410 \\
p<0,001\end{array}$ & 1 & & $\begin{array}{l}r=0,048 \\
\mathrm{p}=0,622\end{array}$ \\
\hline Tempo de uso de Fintech & $\begin{array}{l}r=0,225 \\
p=0,049\end{array}$ & $\begin{array}{l}r=0,048 \\
\mathrm{p}=0,679\end{array}$ & 1 & $\begin{array}{l}r=-0,130 \\
\mathrm{p}=0,260\end{array}$ \\
\hline $\begin{array}{l}\text { Expectativa de } \\
\text { Desempenho }\end{array}$ & $\begin{array}{c}r=-0,061 \\
p=0,534\end{array}$ & $\begin{array}{l}r=0,063 \\
\mathrm{p}=0,519\end{array}$ & $\begin{array}{l}r=-0,069 \\
p=0,549\end{array}$ & $\begin{array}{l}r=0,700 \\
p<0,001\end{array}$ \\
\hline Expectativa de Esforço & $\begin{array}{l}r=-0,105 \\
p=0,282\end{array}$ & $\begin{array}{l}r=0,115 \\
\mathrm{p}=0,238\end{array}$ & $\begin{array}{l}r=-0,166 \\
p=0,150\end{array}$ & $\begin{array}{l}r=0,674 \\
\mathrm{p}<0,001\end{array}$ \\
\hline Influência Social & $\begin{array}{l}r=0,026 \\
p=0,791\end{array}$ & $\begin{array}{l}r=0,077 \\
\mathrm{p}=0,430\end{array}$ & $\begin{array}{c}r=-0,169 \\
\mathrm{p}=0,142\end{array}$ & $\begin{array}{l}r=0,446 \\
p<0,001\end{array}$ \\
\hline Condições Facilitadoras & $\begin{array}{c}r=-0,023 \\
p=0,816\end{array}$ & $\begin{array}{l}r=0,169 \\
p=0,083\end{array}$ & $\begin{array}{l}r=-0,092 \\
\mathrm{p}=0,429\end{array}$ & $\begin{array}{l}r=0,618 \\
\mathrm{p}<0,001\end{array}$ \\
\hline Motivação Hedônica & $\begin{array}{l}r=0,015 \\
p=0,876\end{array}$ & $\begin{array}{l}r=0,193 \\
p=0,047\end{array}$ & $\begin{array}{l}r=-0,067 \\
\mathrm{p}=0,561\end{array}$ & $\begin{array}{l}r=0,663 \\
\mathrm{p}<0,001\end{array}$ \\
\hline Valor de Preço & $\begin{array}{c}r=-0,114 \\
p=0,243\end{array}$ & $\begin{array}{l}r=0,091 \\
\mathrm{p}=0,352\end{array}$ & $\begin{array}{l}r=-0,219 \\
p=0,055\end{array}$ & $\begin{array}{l}r=0,737 \\
p<0,001\end{array}$ \\
\hline Hábito & $\begin{array}{c}r=-0,170 \\
\mathrm{p}=0,081\end{array}$ & $\begin{array}{l}r=0,117 \\
\mathrm{p}=0,233\end{array}$ & $\begin{array}{l}r=-0,111 \\
\mathrm{p}=0,337\end{array}$ & $\begin{array}{l}r=0,741 \\
p<0,001\end{array}$ \\
\hline Inovatividade & $\begin{array}{c}r=-0,185 \\
\mathrm{p}=0,058\end{array}$ & $\begin{array}{l}r=0,117 \\
p=0,234\end{array}$ & $\begin{array}{l}r=-0,143 \\
p=0,214\end{array}$ & $\begin{array}{l}r=0,504 \\
p<0,001\end{array}$ \\
\hline Risco Percebido & $\begin{array}{l}r=0,144 \\
p=0,141\end{array}$ & $\begin{array}{l}r=-0,032 \\
\mathrm{p}=0,742\end{array}$ & $\begin{array}{l}r=-0,005 \\
\mathrm{p}=0,967\end{array}$ & $\begin{array}{l}r=-0,110 \\
\mathrm{p}=0,263\end{array}$ \\
\hline Confiança & $\begin{array}{c}r=-0,088 \\
\mathrm{p}=0,371\end{array}$ & $\begin{array}{l}r=0,230 \\
p=0,018\end{array}$ & $\begin{array}{l}r=-0,010 \\
\mathrm{p}=0,933\end{array}$ & $\begin{array}{l}r=0,586 \\
p<0,001\end{array}$ \\
\hline
\end{tabular}

Nota. Fonte: Elaborada pelos autores (2020).

Em seguida foi realizada uma correlação de Spearman a fim de identificar se existem relações entre as variáveis escolaridade, frequência de uso de fintech, frequência de compra online, tempo de uso de internet e frequência de uso de internet e os fatores analisados. Como observa-se na tabela 3 , todas as correlações foram fracas ou moderadas. Com relação à variável escolaridade, observaram-se correlações fracas com: frequência de compra online $(r=0,23 ; \mathrm{p}=0,021)$, tempo de uso de internet $(r$ $=0,19 ; \mathrm{p}=0,046)$, condições facilitadoras $(r=0,27 ; \mathrm{p}=0,005)$ e confiança $(r=0,24 ; \mathrm{p}=0,014)$. Esses resultados demonstram que quanto maior o grau de escolaridade dos participantes dessa amostra 
maior a frequência de compra online, maior o tempo de uso de internet, maior a percepção de condições facilitadoras e de confiança que o indivíduo tem sobre o uso de fintech.

Com relação à frequência de uso de fintech observaram-se correlações fracas com: frequência de uso de internet $(r=0,26 ; \mathrm{p}=0,024)$, expectativa de desempenho $(r=0,23 ; \mathrm{p}=0,045)$, expectativa de esforço $(r=0,31 ; \mathrm{p}=0,006)$, condições facilitadoras $(r=0,27 ; \mathrm{p}=0,018)$, motivação hedônica $(r=$ $0,26 ; \mathrm{p}=0,025)$, valor de preço $(r=0,24 ; \mathrm{p}=0,036)$, hábito $(r=0,31 ; \mathrm{p}=0,006)$ e intenção de uso de fintech $(r=0,36 ; \mathrm{p}=0,001)$. Assim, a quanto maior a frequência de uso de uso de fintech, maior a expectativa de que esta irá potencializar o desempenho de tarefas, maior a facilidade e as condições facilitadoras que o indivíduo tem ao usar fintech, maior o prazer e diversão associados ao uso de fintech, maior o custo-benefício percebido, maior o hábito em usar fintech e também a intenção de usála. Pessoas que utilizam internet com maior frequência também tendem a utilizar fintech com mais frequência. Esses resultados demonstram que dentre os usuários que já utilizam fintech, principalmente os que utilizam com maior frequência, têm uma percepção positiva sobre a mesma.

A frequência de compra online obteve correlações com: expectativa de esforço $(r=0,31 ; \mathrm{p}=$ $0,002)$, condições facilitadoras $(r=0,40 ; \mathrm{p}<0,001)$, valor de preço $(r=0,34 ; \mathrm{p}<0,001)$, hábito $(r=$ $0,23 ; \mathrm{p}=0,022)$, intenção de uso de fintech $(r=0,30 ; \mathrm{p}=0,002)$, inovatividade $(r=0,31 ; \mathrm{p}=0,002)$, risco percebido $(r=-0,21 ; \mathrm{p}=0,033)$ e confiança $(r=0,25 ; \mathrm{p}=0,011)$. Ou seja, quanto mais o indivíduo compra online maior a facilidade em usar fintech e possui mais condições facilitadoras, maior o custo-benefício percebido, maior a intenção de usar fintech, maior a probabilidade de utilizar novas tecnologias e outras fintechs, menor o risco percebido em usar fintech e maior a confiança.

0 tempo de uso de internet obteve correlações com: expectativa de desempenho $(r=0,22 ; \mathrm{p}=$ $0,027)$, expectativa de esforço $(r=0,30 ; \mathrm{p}=0,002)$, influência social $(r=0,20 ; \mathrm{p}=0,039)$, condições facilitadoras $(r=0,33 ; \mathrm{p}<0,001)$, motivação hedônica $(r=0,26 ; \mathrm{p}=0,006)$ e valor de preço $(r=0,21 ; \mathrm{p}$ $=0,028)$. Tais resultados demonstram que quanto maior o tempo que o indivíduo usa internet maior será a expectativa de que a fintech irá potencializar o desempenho de suas atividades, maior a facilidade em utilizá-la, maior a influência social exercida sobre o uso de fintech, mais condições facilitadoras de usar fintech o indivíduo percebe, maior o prazer e a diversão associados ao uso de fintech e maior o custo-benefício percebido. Ou seja, quanto mais o usuário tem experiência em utilizar internet maior a percepção desses fatores com relação ao uso de fintech.

Com relação à frequência de uso de internet por dia, observou-se correlações baixas com: expectativa de esforço $(r=0,26 ; \mathrm{p}=0,007)$, condições facilitadoras $(r=0,22 ; \mathrm{p}=0,023)$, valor de preço $(r=0,26 ; \mathrm{p}=0,007)$, intenção de uso de fintech $(r=0,23 ; \mathrm{p}=0,016)$, confiança $(r=0,26 ; \mathrm{p}=0,006)$. Quanto mais o indivíduo utiliza internet maior será a facilidade em usar fintech, maior o custobenefício em usá-la e também maior será a intenção de uso. A tabela 3 apresenta todas as correlações encontradas destacadas.

Tabela 3

Correlação de Spearman

\begin{tabular}{ccccc} 
Escolaridade & $\begin{array}{c}\text { Frequência } \\
\text { de uso de } \\
\text { Fintech }\end{array}$ & $\begin{array}{c}\text { Frequência } \\
\text { de compra } \\
\text { online }\end{array}$ & $\begin{array}{c}\text { Tempo de } \\
\text { uso de } \\
\text { internet }\end{array}$ & $\begin{array}{c}\text { Frequência } \\
\text { de uso de } \\
\text { internet }\end{array}$ \\
\hline
\end{tabular}

Escolaridade

Frequência de uso de Fintech

Frequência de compra online

Tempo de uso de internet

Frequência de uso de internet

Expectativa de

Desempenho

Expectativa de

\section{1}

$\begin{array}{ll}r=0,026 & 1 \\ \mathrm{p}=0,823 & r=0,205 \\ r=0,229 & \mathrm{p}=0,073 \\ \mathrm{p}=0,021 & r=0,038 \\ r=0,194 & \mathrm{p}=0,744 \\ \mathrm{p}=0,046 & r=0,257 \\ r=0,139 & \mathrm{p}=0,024 \\ \mathrm{p}=0,154 & r=0,230 \\ r=0,118 & \mathrm{p}=0,045 \\ \mathrm{p}=0,228 & r=0,311 \\ r=0,155 & \end{array}$

\section{1}

$r=0,062$

$\mathrm{p}=0,534$

$r=0,155$

$\mathrm{p}=0,119$

$r=0,129$

$\mathrm{p}=0,197$

$r=0,306$
1

$r=0,141$

$\mathrm{p}=0,150$

$r=0,215$

$\mathrm{p}=0,027$

$r=0,301$
1

$r=0,122$

$\mathrm{p}=0,212$

$r=0,259$ 


\begin{tabular}{cccccc} 
Esforço & $\mathrm{p}=0,113$ & $\mathrm{p}=0,006$ & $\mathrm{p}=0,002$ & $\mathrm{p}=0,002$ & $\mathrm{p}=0,007$ \\
Influência Social & $r=0,111$ & $r=0,118$ & $r=-0,016$ & $r=0,200$ & $r=0,128$ \\
Condições & $\mathrm{p}=0,256$ & $\mathrm{p}=0,308$ & $\mathrm{p}=0,876$ & $\mathrm{p}=0,039$ & $\mathrm{p}=0,191$ \\
Facilitadoras & $r=0,272$ & $r=0,270$ & $r=0,402$ & $r=0,329$ & $r=0,221$ \\
Motivação Hedônica & $\mathrm{p}=0,005$ & $\mathrm{p}=0,018$ & $\mathrm{p}<0,001$ & $\mathrm{p}<0,001$ & $\mathrm{p}=0,023$ \\
& $\mathrm{p}=0,186$ & $r=0,255$ & $r=0,160$ & $r=0,263$ & $r=0,070$ \\
Valor de Preço & $r=0,186$ & $\mathrm{p}=0,025$ & $\mathrm{p}=0,108$ & $\mathrm{p}=0,006$ & $\mathrm{p}=0,476$ \\
& $\mathrm{p}=0,057$ & $\mathrm{p}=0,036$ & $\mathrm{p}<0,001$ & $\mathrm{p}=0,028$ & $\mathrm{p}=0,007$ \\
Hábito & $r=0,118$ & $r=0,313$ & $r=0,226$ & $r=0,020$ & $r=0,175$ \\
Intenção de uso de & $\mathrm{p}=0,229$ & $\mathrm{p}=0,006$ & $\mathrm{p}=0,022$ & $\mathrm{p}=0,841$ & $\mathrm{p}=0,073$ \\
fintech & $r=0,151$ & $r=0,357$ & $r=0,304$ & $r=0,171$ & $r=0,234$ \\
Inovatividade & $\mathrm{p}=0,123$ & $\mathrm{p}=0,001$ & $\mathrm{p}=0,002$ & $\mathrm{p}=0,080$ & $\mathrm{p}=0,016$ \\
& $r=0,089$ & $r=0,160$ & $r=0,307$ & $r=0,096$ & $r=0,170$ \\
Risco Percebido & $\mathrm{p}=0,364$ & $\mathrm{p}=0,165$ & $\mathrm{p}=0,002$ & $\mathrm{p}=0,328$ & $\mathrm{p}=0,102$ \\
& $r=-0,018$ & $r=-0,108$ & $r=-0,211$ & $r=-0,068$ & $r=-0,110$ \\
Confiança & $\mathrm{p}=0,853$ & $\mathrm{p}=0,351$ & $\mathrm{p}=0,033$ & $\mathrm{p}=0,487$ & $\mathrm{p}=0,263$ \\
\hline
\end{tabular}

Nota. Fonte: Elaborado pelos autores (2020).

Também foi realizado um teste $t$ de Student a fim de identificar se existem diferenças entre os fatores analisados e as variáveis sexo, dispositivos usados, compra online, uso de internet banking e preferência de compra. Com relação ao sexo, apenas o fator valor de preço apresentou diferenças significativas $\left[\mathrm{t}_{(104)}=2,26 ; \mathrm{p}=0,026\right]$. O sexo masculino apresentou deu maior importância ao custobenefício de usar fintech $(\mathrm{m}=5,54 ; \mathrm{dp}=0,17)$ do que o sexo feminino $(\mathrm{m}=5,08 ; \mathrm{dp}=0,16)$. Com relação aos dispositivos utilizados para acessar a internet, observou-se diferenças apenas no fator risco percebido $\left[\mathrm{t}_{(77,88)}=-2,54 ; \mathrm{p}=0,13\right]$, sendo que o grupo que utiliza computador $(\mathrm{m}=4,66 ; \mathrm{dp}=$ $0,22)$ obteve maior percepção de risco do que o grupo que utiliza celular $(\mathrm{m}=3,92 ; \mathrm{dp}=0,19)$. De acordo com esse resultado utilizar o computador para acessar a internet oferece mais riscos para essa amostra.

Observaram-se diferenças entre o grupo que prefere comprar online e presencialmente em todos os fatores, exceto o fator influência social. 0 grupo que prefere comprar online obteve as maiores médias, menos no fator risco percebido, onde o grupo que prefere comprar presencialmente obteve média maior. Esse resultado indica que o risco percebido é maior para os indivíduos que preferem comprar presencialmente. 0 grupo que prefere realizar compra online apresentou maior intenção de usar fintech. Com relação aos usuários e não usuários de internet banking todos os fatores apresentaram diferenças significativas. Exceto no fator risco percebido, as médias foram maiores no grupo que utiliza internet banking. Os não usuários de internet banking apresentam maior percepção de risco que os que usuários.

Foi realizada uma regressão múltipla padrão considerando o fator intenção de uso de fintech como variável critério $\left[\mathrm{F}_{(10,95)}=24,19 ; \mathrm{p}<0,001\right]$ e os fatores como variáveis independentes. De acordo com o quadro 1, as variáveis independentes são: Expectativa de Desempenho, Expectativa de Esforço, Influência Social, Condições Facilitadoras, Motivação Hedônica, Valor de Preço, Hábito, Inovatividade, Risco Percebido e confiança. Os fatores expectativa de esforço, condições facilitadoras e risco percebido apresentaram coeficientes não-padronizados negativos, indicando que existe relação inversa entre estes fatores a intenção de usar fintech. Os preditores apresentaram 69\% de variância explicada para a intenção de uso de fintech. Os preditores significativos foram expectativa de desempenho, valor de preço e hábito, sendo o valor de preço o fator que mais contribuiu para explicar a intenção de usar fintech. Os demais fatores não colaboraram para explicar a intenção de uso de fintech nessa análise de regressão. 
Tabela 4

Regressão múltipla padrão com fatores

\begin{tabular}{|c|c|c|c|c|c|c|c|c|c|}
\hline Base & $\mathbf{R}$ & $\mathbf{R}^{2}$ & $\begin{array}{c}\mathbf{R}^{2} \\
\text { ajustado }\end{array}$ & $\begin{array}{c}\text { Erro } \\
\text { padrão }\end{array}$ & Preditores & B & $\boldsymbol{\beta}$ & $\mathbf{t}$ & $\mathbf{p}$ \\
\hline \multirow{3}{*}{$\begin{array}{l}\text { Intenção } \\
\text { de uso de } \\
\text { Fintech }\end{array}$} & \multirow{3}{*}{0,85} & \multirow{3}{*}{0,72} & \multirow{3}{*}{0,69} & \multirow{3}{*}{0,73} & $\begin{array}{c}\text { Expectativa } \\
\text { de } \\
\text { Desempenho }\end{array}$ & ,423 & ,314 & ,215 & $=0,002$ \\
\hline & & & & & $\begin{array}{l}\text { Valor de } \\
\text { Preço }\end{array}$ & ,437 & ,408 & ,458 & $<0,001$ \\
\hline & & & & & Hábito & ,326 & ,279 & ,023 & $=0,003$ \\
\hline
\end{tabular}

Nota. Fonte: Elaborado pelos autores (2020).

Foi realizada também uma regressão múltipla padrão considerando o fator intenção de uso de fintech como variável critério $\left[\mathrm{F}_{(8,97)}=7,66 ; \mathrm{p}<0,001\right]$, e as variáveis sociodemográficas e comportamentais (sexo, idade, renda individual mensal, escolaridade, frequência de uso de fintech , frequência de compra online, tempo de uso de internet e frequência de uso de internet) como variáveis independentes. A variável idade apresentou coeficiente não-padronizado negativo, indicando que existe relação inversa entre idade e a intenção de usar fintech. Os preditores apresentaram 34\% de variância explicada para a intenção de uso de fintech. Os preditores significativos foram idade, compra online e usa internet banking, sendo a compra online a variável que mais contribuiu para explicar a intenção de usar fintech. Os demais fatores não colaboraram para explicar a intenção de uso de fintech nessa análise de regressão.

Tabela 5

Regressão múltipla padrão com variáveis sociodemográficas e comportamentais

\begin{tabular}{|c|c|c|c|c|c|c|c|c|c|}
\hline Base & $\mathbf{R}$ & $\mathbf{R}^{2}$ & $\begin{array}{c}\mathbf{R}^{2} \\
\text { ajustado }\end{array}$ & $\begin{array}{c}\text { Erro } \\
\text { padrão }\end{array}$ & Preditores & B & $\boldsymbol{\beta}$ & $\mathbf{T}$ & $\mathbf{p}$ \\
\hline \multirow{3}{*}{$\begin{array}{l}\text { Intenção } \\
\text { de uso de } \\
\text { Fintech }\end{array}$} & \multirow{3}{*}{0,62} & \multirow{3}{*}{0,39} & \multirow{3}{*}{0,34} & \multirow{3}{*}{1,06} & Idade &,- 049 &,- 255 & $-2,74$ & $=0,007$ \\
\hline & & & & & $\begin{array}{l}\text { Compra } \\
\text { online }\end{array}$ & ,977 & 291 & 131 & $=0,002$ \\
\hline & & & & & $\begin{array}{l}\text { Internet } \\
\text { banking }\end{array}$ & ,639 & , 185 & ,957 & $=0,05$ \\
\hline
\end{tabular}

Nota. Fonte: Elaborado pelos autores (2020).

Dessa forma, apenas expectativa de desempenho, valor de preço e hábito afetaram a intenção de usar fintech, indicando que quanto maiores forem a expectativa de potencializar o desempenho em atividades, o custo-benefício percebido e o hábito que o indivíduo possui maior será a intenção de usar fintech.

No trabalho de Zeltser, Maçada e Mallmann (2017) a utilidade percebida do uso de fintech impactou positivamente no uso de fintech. Esse construto, que faz parte do modelo TAM - Technology Acceptance Model (Davis, 1989), assemelha-se em sua definição ao construto expectativa de desempenho utilizado nesse trabalho, onde o indivíduo acredita que o uso de fintech irá potencializar o seu desempenho em atividades. Assim, esse resultado corrobora os achados nesse trabalho. 0 estudo de Narteh, Mahmoud e Amoh (2017), que utilizou o modelo TAM como base, encontrou forte influência da facilidade de uso percebida, utilidade percebida, confiança percebida e o custo percebido no uso do serviço de dinheiro móvel. Ao contrário dos resultados encontrados no presente estudo, a pesquisa de Narteh et al. (2017) encontrou influência da confiança percebida na intenção de usar o serviço.

O estudo de Senyo e Osabutey (2020) também encontrou forte relação entre a intenção de usar serviços de dinheiro móvel e a expectativa de desempenho. Ao utilizar o modelo UTAU2, as variáveis valor de preço, motivação hedônica e influência social não apresentaram influência na intenção de uso desses serviços. 0 risco percebido também foi analisado nessa pesquisa e não apresentou influência, bem como no presente estudo. A presença desses resultados semelhantes sugere que os usuários de fintechs estão mais preocupados com as facilidades e o desempenho melhorado que terão ao usar os 
serviços, em comparação com os serviços tradicionais de banco, do que na percepção do risco, na diversão, ou até mesmo na influência dos seus pares para utilizá-los.

\section{CONSIDERAÇÕES FINAIS}

Esse trabalho teve como objetivo analisar a intenção de uso de fintechs através das variáveis do modelo UTAUT 2 de Venkatesh et al. (2012), com adição das variáveis propostas por Slade et al. (2015). De acordo com os resultados encontrados na análise de regressão realizada apenas expectativa de desempenho, valor de preço e hábito afetaram a intenção de uso de fintech nessa amostra.

Ao acrescentar as variáveis propostas por Slade et al. (2015) - inovatividade, risco percebido e confiança - esperava-se que estas afetassem a intenção de uso de fintech, o que não ocorreu. No entanto, é importante considerar que a amostra utilizada é composta em sua maioria por pessoas com alto grau de instrução, jovens e que possuem experiência com internet, o que pode ter influenciado os resultados. Com relação às variáveis do modelo de Venkatesh et al. (2012) apenas a expectativa de desempenho, valor de preço e hábito afetaram a intenção de uso de fintech, o que apresenta algumas implicações gerenciais.

Considerando que os consumidores e potenciais consumidores dão importância a estas variáveis as empresas de fintech podem investir no aprimoramento das suas funcionalidades, a fim de aumentar a expectativa de desempenho, o que consequentemente aumentará a intenção de uso de fintech, conforme os resultados encontrados nessa pesquisa. Podem também melhorar a relação custobenefício de usar fintech, visto que o valor de preço foi um importante preditor da intenção de uso, onde as fintechs surgem como alternativa às empresas tradicionais do mercado financeiro, tendo como principal vantagem para o consumidor taxas menores. Quanto mais os consumidores e potenciais consumidores perceberem que terão benefícios a um custo inferior do que as empresas tradicionais oferecem, mais utilizarão fintechs. 0 hábito de utilizar fintech também pode ser influenciado pelas empresas, investindo em ações de marketing, dando incentivos aos consumidores e gerenciando o seu relacionamento com esses, a fim de estimular o uso de fintech, que ao tornar-se um hábito, aumenta a probabilidade de usá-la, ou seja, quanto mais se usa fintech, mais pretende-se usar.

Como os resultados indicam, quanto menor a idade maior a intenção de usar fintech. Assim, as empresas devem propor incentivos ao público de mais idade, a fim de atender suas necessidades e estimulá-los a usar fintech, conquistando também esses consumidores. Também merecem atenção as pessoas que possuem o hábito de comprar online e que utilizam internet banking, visto que estas apresentaram maior intenção de usar fintech. Desse modo, as empresas podem investir em propaganda no meio digital, em sites de varejo online, estimulando o uso de fintech. Vale ressaltar que os resultados encontrados nesse trabalho encontram limitações: a amostra considerada pequena e não probabilística não permite que os resultados sejam generalizados para a população. Dada a impossibilidade de conhecer todos os participantes da população, torna-se inviável utilizar uma amostragem probabilística. Por fim, recomenda-se que estudos futuros realizem pesquisa utilizando outros modelos de aceitação e tecnologia, bem como sejam propostas novas variáveis para analisar o comportamento de uso de fintechs.

\section{References}

Araujo, M. V. (2018). Investimento em tecnologia nas instituições financeiras e a influência das fintechs. Dissertação (Mestrado em Economia) - Fundação Getúlio Vargas - FGV.

Arner, D. W., Barberis, J., \& Buckley, R. P. (2015). The evolution of Fintech: A new post-crisis paradigm. Geo. J. Int'l L., 47, 1271.

Banco Mundial (2018). Financial inclusion on the rise, but gaps remain. Recuperado de: https://www.worldbank.org/en/news/press-release/2018/04/19/financial-inclusion-on-therise-but-gaps-remain-global-findex-database-shows. 
Baganzi, R., \& Lau, A. K. (2017). Examining trust and risk in mobile money acceptance in Uganda. Sustainability, 9(12), 2233.

Ciab Febraban (2017). Bancos $x$ Fintechs ou Bancos $e$ Fintechs? Recuperado de: http://www.ciab.org.br/publicacoes/edicao/66/bancos-x-fintechs-ou-bancos-e-fintechs

Cernev, A. K. (2010). Mobile banking no brasil: Eventos Críticos, Trajetória e Cenários Esperados, São Paulo-SP.

Chihimi, K. A. (2018). Fintech \& The Banking Industry: Disruption or Evolution? Dissertação (Mestrado Profissional em Gestão Internacional) - Fundação Getúlio Vargas - FGV, São Paulo.

Conexão Fintech (2017). O que é Fintech? Recuperado de: http://conexaofintech.com.br/fintech/oque-e-fintech/

Davis, F. D. (1989). Perceived usefulness, perceived ease of use, and user acceptance of information technology. MIS quarterly, 319-340.

Ferreira, C. A., Junior, P. S. P., Silva, S. W., \& Portugal, N. S. (2017). Novas evoluções do mercado de crédito: Uma análise sobre as Fintechs. Anais do VI SINGEP, São Paulo - SP.

Finnovation (2018). O que é Fintech. Recuperado de: http://finnovation.com.br/

Guidi, C. E. G. (2018). Análise da segmentação aplicada à aquisição de clientes no setor de crédito pessoal. Dissertação (Mestrado em Gestão para a Competitividade) - Fundação Getúlio Vargas FGV.

Likert, R. (1932). A technique for the measurement of attitudes. Archives of psychology.

Lisboa, E., \& Mendonça, A. V. B. F. (2017). As vantagens e desvantagens de ser um clientr Nubank. XXVIII ENANGRAD. Brasília.

Marcon, R. (2018). O centro de serviços compartilhados na instituição financeira digital: Uma análise das fintechs e do impacto nas atuais estruturas de bankoffice. Dissertação (Mestrado Profissional em Gestão e Negócios) - Universidade do Vale do Rio dos Sinos - UNISINOS.

Ministério da Fazenda (2016). Cartilha de promoção à concorrência. 2016. Recuperado de: http://www.fazenda.gov.br/centrais-de-conteudos/publicacoes/cartilhas/arquivos/cartilhapromocao-a-concorrencia.pdf

Miranda, M. B. (2011). 0 Monopólio e o Oligopólio. Revista Virtual Direito Brasil. V. 5 - no 2, 2011.

Narteh, B., Mahmoud, M. A., \& Amoh, S. (2017). Customer behavioural intentions towards mobile money services adoption in Ghana. The Service Industries Journal, 37(7-8), 426-447.

Osei-Assibey, E. (2015). What drives behavioral intention of mobile money adoption? The case of ancient susu saving operations in Ghana. International Journal of Social Economics.

Rodrigues, L. A. (2012). Tecnologia voltada para o cliente. Revista CIAB FEBRABAN.

Sanches, C., Meireles, M., \& Sordi, J. O. (2011). Análise Qualitativa Por Meio da Lógica Paraconsistente: Método de Interpretação e Síntese de Informação obtida Por Escalas Likert. Anais do III Encontro de Ensino e Pesquisa em Administração e Contabilidade, João Pessoa-PB.

Senyo, P. K., \& Osabutey, E. L. (2020). Unearthing antecedents to financial inclusion through FinTech innovations. Technovation, 98, 102155.

Slade, E. L., Dwivedi, Y. K., Piercy, N. C., \& Williams, M. D. (2015). Modeling consumers' adoption intentions of remote mobile payments in the United Kingdom: extending UTAUT with innovativeness, risk, and trust. Psychology \& Marketing, 32(8), 860-873.

Venkatesh, V., Thong, J. Y., \& Xu, X. (2012). Consumer acceptance and use of information technology: extending the unified theory of acceptance and use of technology. MIS Quarterly, 157-178.

Zeltser, T., Maçada, A., \& Mallmann, G. (2017). Efeitos da Qualidade da Informação e da Qualidade do Serviço da Informação no Uso de Serviços de Fintech. SEMEAD XX-Anais. 